\title{
Clinical characteristics and mortality of patients in the intensive care unit with and without new-onset arrhythmias
}

\author{
ZHANG YONG-LI' $I^{1}$ HAI FENG ${ }^{2}$, YANG YAN-ZONG ${ }^{3}$ \\ ${ }^{1}$ First Affiliated Hospital of Dalian Medical University, Department of Intensive Care Medicine, Liaoning, China \\ ${ }^{2}$ Third People's Hospital of Dalian, Department of Intensive Care Medicine, Liaoning, China \\ ${ }^{3}$ First Affiliated Hospital of Dalian Medical University, Department of Cardiology, Liaoning, China
}

Corresponding author

Yang Yan-Zong

Department of Cardiology,

First Affiliated Hospital of Dalian Medical University

Zhongshan Road 222, DaLian, Liaoning, China (116011)

Phone: 008613500784272

E-mail: yyzheart@163.com

\section{ABSTRACT}

Objective. The aims of this study were to analyze the risk factors for and types of new-onset arrhythmias in ICU (Intensive care unit), and to evaluate their impact on patient outcomes.

Methods. We studied 1051 patients who were admitted to the two general ICUs between December 2013 and February 2016. These patients were divided into two groups: patients with new-onset arrhythmias and patients without new-onset arrhythmias. We compared the risk factors, types and prognoses of new-onset arrhythmias between these two groups.

Results. New-onset arrhythmias were observed in $20.84 \%(n=219)$ of 1051 patients. The main risk factors leading to arrhythmias included age, emergency operation, past cardiovascular disease, patients with multiple systemic diseases, acute respiratory distress syndrome, severe sepsis/septic shock, acute renal dysfunction, cardiovascular disease, electrolyte disturbance, patients on ventilators, patients on vasopressors and higher Acute Physiology and Chronic Health Evaluation II scores (APACHE II score) on ICU admission. Multivariate logistic regression revealed that age, emergency operation, severe sepsis/septic shock, cardiovascular disease, electrolyte disturbance, patients on ventilators and those with higher APACHE II scores on ICU admission, were all significantly associated with new-onset arrhythmias. Arial fibrillation was the most frequent arrhythmia. ICU mortality in patients with newonset arrhythmias was $22.37 \%$ (49 out of 219 ) compared with $3.61 \%$ (30 out of 832 ) in patients without new-onset arrhythmias $(\mathrm{p}<0.001)$. Among surviving patients, ICU stay for those with new-onset arrhythmias was longer than those without new-onset arrhythmias (median stay of 10 days versus 5 days, $\mathrm{p}<0.001)$.

Conclusion. We found a high prevalence of new-onset arrhythmias in ICU patients. Arrhythmia, especially atrial fibrillation, was a common complication in ICU patients and was associated with increasing length of ICU stay and higher mortality.

Key words: arrhythmia, intensive care unit, critical illness, mortality

\section{INTRODUCTION}

Arrhythmias are associated with an unfavorable prognosis, increasing the risk of death. Although arrhythmias are traditionally associated with cardiovascular diseases, there is evidence of a high incidence of arrhythmias in patients with noncardiovascular diseases, such as sepsis, chronic obstructive pulmonary disease, acute renal dysfunction. Arrhythmias are frequently observed in critically ill patients, and studies about arrhythmias in intensive care units (ICU) have been reported. (1-3) All types of arrhythmias can be observed, but atrial arrhythmias are the most common. (4) In recent years, increasing attention has been paid to atrial fibrillation in septic patients. (5-8) However, there are few studies on the various kinds of patients admitted to a general ICU. Epidemiological insights may help to identify strategies for arrhythmia prevention, monitoring, or risk modification. To better define the burden of arrhythmias in ICU, we analyzed the risk factors, types and prognosis of new-onset arrhythmias in the general ICU population.

\section{MATERIALS AND METHODS}

This study was carried out in two general ICUs: the Department of Intensive Care Medicine of the First Affiliated Hospital of Dalian Medical University and The Third People's Hospital of Dalian. Patients were recruited between December 2013 and February 2016. Those who were in the final stage of their chronic disease, who were awaiting cardiac surgery, or were discharged from ICU within 24 hours were excluded from further analysis.

This study was approved by the Ethics Committees of Dalian Medical University and The Third People's Hospital of Dalian. All procedures were performed in accordance with the Declaration of Helsinki. Informed consent was obtained from either the patient (if competent) or his/her family.

\section{Data collection}

During ICU stay, all patients underwent continuous electrocardiographic monitoring and automatic detection of arrhythmias. All episodes of arrhythmia were recorded. The following outcomes were assessed: (a) sustained tachyarrhythmia lasting more than 30 seconds; (b) tachyarrhythmia requiring therapy; (c) bradycardia of less than 40 beats/ min or requiring intervention; (9) (d) in the case of chronic arrhythmias, only new arrhythmias were considered. We recorded the date of ar- 
rhythmias and the types of arrhythmias. At least three attending doctors retrospectively analyzed new-onset arrhythmias and then determined the most likely causes and inducing factors.

\section{Statistical analysis}

Data were analyzed using SPSS version 17.0 statistical software. Baseline data such as age, Acute Physiology and Chronic Health Evaluation II score (APACHE II score) and ICU length of stay was expressed as mean \pm standard deviation (SD) and analyzed using student's t test. Qualitative data were presented as frequencies and analyzed using the Pearson chi-square test. Multivariate logistic regression analysis was performed to determine the most significant risk factors. Survival rates were analyzed using the Kaplan-Meier method and were compared using the logrank test. A value of $p<0.05$ was considered statistically significant in all analyses.

\section{RESULTS}

\section{Risk factors for arrhythmias}

Among 1051 patients, during this 26-month study, 20.84\% ( $\mathrm{n}=219)$ experienced new-onset arrhythmias, while $79.16 \%(n=832)$ were those without newonset arrhythmias. Univariate analysis was performed on factors impacting new-onset arrhythmias, based on medical records, as shown in Table 1. In the new-onset group there were 142 males (64.84\%) and $77 \mathrm{fe}$ males $(35.16 \%)$, with an average age of $70.42 \pm 14.99$ years, while in the group without new-onset arrhythmias, there were 538 males (64.66\%) and 294 females (35.33\%), with an average age of $64.38 \pm 14.77$ years. These data suggest that there was a significant difference in age $(\mathrm{p}<0.001)$, but not in gender $(\mathrm{p}=0.961)$, between the groups with or without new-onset arrhythmias.

Surgical patients, either for emergency operations or elective operations, accounted for $37.68 \%(n=396)$ of cases,. As shown in Table 1, there was a statistically significant difference between the two groups when it came to emergency operations $(\mathrm{p}<0.001)$. It was obvious that the patients with a history of cardiovascular disease had an increased incidence of new-onset arrhythmias compared to those without a history of cardiovascular disease $(\mathrm{p}<0.001)$. Patients with multiple system diseases were more prone to arrhythmias; however, patients with a history of pulmonary disease, endocrine disease and kidney disease did not show any difference between the groups ( $\mathrm{p}>0.05)$. There were more patients with acute respiratory distress syndrome (ARDS), severe sepsis/ septic shock, acute renal dysfunction and cardiovascular disease in the group of new-onset arrhythmias than that without new-onset arrhythmias. In addition, a higher percentage of patients in the group of new-onset arrhythmias received mechanical ventilation for more than 24 hours and used vasopressors than those without new-onset arrhythmias $(\mathrm{p}<0.001)$. A higher percentage of electrolyte disturbance was also observed in the group of new-onset arrhythmias ( $\mathrm{p}=$ 0.005). During the ICU stay, as shown in Table 1, the patients in the group of newonset arrhythmias had higher APACHE II scores than those without new-onset arrhythmias, $19.86 \pm 6.13$ versus $16.03 \pm 5.83$ $(\mathrm{p}<0.001)$.

Multivariate logistic regression revealed that age, emergency operation, severe sepsis/septic shock, cardiovascular disease, electrolyte disturbance, patients on ventilators and higher APACHE II scores on ICU admission, were all significantly associated with new-onset arrhythmias (Table 2).

\section{Arrhythmia types in ICU}

As shown in Table 3, atrial arrhythmias, ventricular arrhythmias and conduction abnormalities were recorded. There were 266 arrhythmia episodes in 219 patients with new-onset arrhythmias, which included 150 episodes of atrial arrhythmias in 120 patients, 68 episodes of ventricular arrhythmias in 54 patients and 48 episodes of conduction abnormalities in 37 patients. The average episode time for patients with new-onset arrhythmias was $20.33 \pm 18.51$ hours after ICU admission. New-onset arrhythmia is more likely to occur within the first 48 hours of ICU admission.

\section{Patient prognosis}

As shown in Table 4, ICU mortality rate in patients with new-onset arrhythmias was $22.37 \%$ (49 out of 219), which was statistically significantly higher than in those without new-onset arrhythmias, 3.61\% (37 out of 832) $(\mathrm{p}<0.001)$. The difference in 28-day mortality rate was also statistically significant between these two groups ( $\mathrm{p}<0.001$ ). Kaplan-Meier survival curves are shown in Figure 1, according to the 28-day mortality rate for patients with or without new-onset arrhythmias. Among the surviving patients, the ones with newonset arrhythmias had a longer ICU stay, a median of 10 days compared to 5 days in those without new-onset arrhythmias (11.70 \pm 10.11 versus7.94 $\pm 7.75, p<0.001)$. The median, minimum, maximum and interquartile range (box) are shown in Figure 2.

\section{DISCUSSION}

In our study we reported a high prevalence of new-onset arrhythmias (20.84\%) in ICU patients, similar to findings in other studies. (10) Arrhythmias in critically ill patients are a specific risk factor for prognosis and an indication of severity of illness. Most of the studies on arrhythmias have revealed that arrhythmias increase the length of stay in hospital and are markers of poor prognosis. $(8,11)$ As expected, in our study the occurrence of new-onset arrhythmias during critical illness was associated with a poor prognosis; patients with new-onset arrhythmias exhibited a trend towards longer ICU stay and higher mortality rates (Figures 1,2).

APACHE II scores have been widely adopted to evaluate the severity of illness. In our study, a higher APACHE II score in patients with new-onset arrhythmias suggested there was an association between severity of illness and the occurrence of arrhythmias. A higher percentage of mechanical ventilation for more than 24 hours and vasopressors usage at ICU admission were also found in the new-onset arrhythmia group.

It is clear that during critical illness patients with cardiac disease tend to be at risk for arrhythmias and sudden death. In our study, patients with multiple system diseases were more likely to experience arrhythmias. Although most of our patients $(87.06 \%)$ were admitted for non-cardiac disease, $10.28 \%$ of all patients and $16.35 \%$ of patients in the arrhythmia group had a cardiovascular history. It was evident that the occurrence of arrhythmias was increased in patients with an underlying disease, especially with heart disease. Thus, these patients deserve more attention. (12) Elderly patients have a higher incidence of arrhythmia; the etiology is multifactorial. Fibrosis and loss of muscle mass in the heart play a major role in the development of arrhythmia. Fuchs et al. analyzed ICU patients above the age of 65 and above the age of 85 and found that there was a very high incidence of cardiac arrhythmias in the group above the age of 85 (24.6 vs. $43.5 \%)$. (13) Therefore, age was associated with long-term mortality, as discussed by others. (14) 
Sepsis is a systemic, deleterious host response to infection leading to severe sepsis and septic shock. Sepsis affects more than 1 million hospitalized patients yearly in United States. (15) Severe sepsis and septic shock are affecting millions of people around the world each year, killing one in four (and often more), and increasing in incidence. (16) Severe sepsis/ septic shock is also the main cause of arrhythmias in the ICU. (17) It has been reported that atrial fibrillation is often found in septic patients. (18) Patients with new-onset atrial fibrillation during severe sepsis have greater risk for in-hospital strokes and increased longterm risks of heart failure, ischemic stroke, and death. (19) Besides severe sepsis/septic shock, ARDS was another risk factor for arrhythmia, especially in patients with heart disease, because of the acidosis and hyperkalemia caused by acute hypoxia.

As shown in Tables 1 and 2, there was a greater association between electrolyte disturbance and arrhythmias. Abnormal electrolyte serum blood levels, such as high serum potassium, hypomagnesium and hypophosphatemia are highly correlated

Table 1. Risk factors for arrhythmias on ICU admission

\begin{tabular}{|c|c|c|c|}
\hline Risk factors & Arrhythmia group (n=219) & Non- arrhythmia group $(n=832)$ & p -value ${ }^{*}$ \\
\hline Age & $70.42 \pm 14.99$ & $64.38 \pm 14.77$ & $<0.001$ \\
\hline Gender (male/female) & $142 / 77(64.84 \% / 35.16 \%)$ & $538 / 294(64.66 \% / 35.34 \%)$ & 0.961 \\
\hline Emergency operation & $44(20.09 \%)$ & $64(7.69 \%)$ & $<0.001$ \\
\hline Elective operation & $71(32.42 \%)$ & $217(26.08 \%)$ & 0.061 \\
\hline \multicolumn{4}{|l|}{ Past medical history } \\
\hline Cardiovascular disease & $38(17.35 \%)$ & $70(8.41 \%)$ & $<0.001$ \\
\hline Pulmonary disease & $34(15.53 \%)$ & $95(11.42 \%)$ & 0.100 \\
\hline Endocrine disease & $27(12.33 \%)$ & $72(8.65 \%)$ & 0.098 \\
\hline Kidney disease & $13(5.94 \%)$ & $33(3.97 \%)$ & 0.205 \\
\hline Patients with multiple systemic diseases & $26(11.87 \%)$ & $59(7.09 \%)$ & 0.021 \\
\hline \multicolumn{4}{|l|}{ Intensive care unit diagnosis } \\
\hline ARDS & $39(17.81 \%)$ & $51(6.13 \%)$ & $<0.001$ \\
\hline Severe sepsis/septic shock & $98(44.75 \%)$ & $136(16.35 \%)$ & $<0.001$ \\
\hline Trauma & $27(12.33 \%)$ & $71(8.53 \%)$ & 0.086 \\
\hline Acute renal dysfunction & $33(15.07 \%)$ & $69(8.29 \%)$ & 0.003 \\
\hline Cardiovascular disease & $55(25.11 \%)$ & $81(0.96 \%)$ & $<0.001$ \\
\hline Pulmonary disease & $53(24.20 \%)$ & $168(20.19 \%)$ & 0.196 \\
\hline Central nervous disease & $24(10.96 \%)$ & $73(8.77 \%)$ & 0.321 \\
\hline Electrolyte disturbance & $105(47.95 \%)$ & $293(35.22 \%)$ & 0.001 \\
\hline Poisoning & $6(2.74 \%)$ & $24(2.88 \%)$ & 0.909 \\
\hline Other acute condition & $12(5.48 \%)$ & $62(7.45 \%)$ & 0.310 \\
\hline Patients on ventilator & $125(57.08 \%)$ & $181(21.75 \%)$ & $<0.001$ \\
\hline Patients on vasopressor & $121(55.25 \%)$ & $176(21.15 \%)$ & $<0.001$ \\
\hline APACHE II score on ICU admission & $19.86 \pm 6.13$ & $16.03 \pm 5.83$ & $<0.001$ \\
\hline
\end{tabular}

ICU, intensive care unit; APACHE II score; Acute Physiology and Chronic Health Evaluation II score; ARDS, acute respiratory distress syndrome.

${ }^{*} \mathrm{p}<0.05$ was defined as indicative of statistical significance

Table 2. Multivariate logistic regression analysis of risk factors for mortality

\begin{tabular}{llll}
\hline Risk factors & Odds ratio & $\mathbf{9 5 \% C I}$ & p -value* \\
\hline Age & 1.031 & $(1.018,1.045)$ & $<0.001$ \\
\hline Emergency operation & 2.648 & $(1.597,4.392)$ & $<0.001$ \\
\hline Severe sepsis / septic shock & 3.358 & $(2.299,4.904)$ & $<0.001$ \\
\hline Cardiovascular disease & 2.439 & $(1.571,3.787)$ & $<0.001$ \\
\hline Electrolyte disturbance & 1.644 & $(1.164,2.322)$ & 0.005 \\
\hline Patients on ventilator & 2.630 & $(1.671,4.138)$ & $<0.001$ \\
\hline APACHE II score on ICU admission & 1.047 & $(1.010,1.085)$ & 0.013 \\
\hline
\end{tabular}

${ }^{*} \mathrm{p}<0.05$ was defined as indicative of statistical significance

ICU, intensive care unit; APACHE II score; Acute Physiology and Chronic Health Evaluation II score. 
Table 3. Arrhythmia types in intensive care unit (ICU) patients

\begin{tabular}{llll}
\hline Arrhythmia type & $\begin{array}{l}\text { Patients with arrhythmias } \\
(\mathbf{n = 2 1 9 )}\end{array}$ & $\begin{array}{l}\text { Episode number of arrhythmias } \\
(\mathbf{n = 2 6 6 )}\end{array}$ & $\begin{array}{l}\text { Average hours } \\
\text { after admission }\end{array}$ \\
\hline Atrial arrhythmias & $120(54.79 \%)$ & $150(56.39 \%)$ & $31.50 \pm 13.75$ \\
\hline Atrial flutter & $25(11.42 \%)$ & $27(10.15 \%)$ & $40.86 \pm 20.20$ \\
\hline Atrial fibrillation & $75(34.25 \%)$ & $86(32.33 \%)$ & $29.11 \pm 6.46$ \\
\hline Paroxysmal atrial tachycardia & $20(9.13 \%)$ & $37(13.91 \%)$ & $14.05 \pm 9.40$ \\
\hline Ventricular arrhythmias & $54(24.66 \%)$ & $68(25.56 \%)$ & $51.48 \pm 25.43$ \\
\hline Ventricular tachycardia & $37(16.89 \%)$ & $39(14.66 \%)$ & $29(10.90 \%)$ \\
\hline Ventricular fibrillation & $17(7.76 \%)$ & $48(18.05 \%)$ & $23.25 \pm 17.05$ \\
\hline Type1 second degree atrioventricular block & $37(16.89 \%)$ & $21(7.89 \%)$ & $20.69 \pm 15.21$ \\
\hline Type2 second degree atrioventricular block & $9(4.11 \%)$ & $18(6.77 \%)$ & $13.55 \pm 7.50$ \\
\hline Third degree atrioventricular block & $11(5.02 \%)$ & $9(3.38 \%)$ & \\
\hline
\end{tabular}

Table 4. Prognosis for patients with or without arrhythmia

\begin{tabular}{llll}
\hline Variables & Arrhythmia group $(\mathbf{n}=\mathbf{2 1 9})$ & Non- arrhythmia group $(\mathbf{n}=\mathbf{8 3 2})$ & $\mathbf{p}$-value \\
\hline ICU mortality & $49(22.37 \%)$ & $30(3.61 \%)$ & $<0.001$ \\
\hline 28-day mortality & $37(16.89 \%)$ & $26(3.13 \%)$ & $<0.001$ \\
\hline ICU length of stay, days (surviving patients) & $\begin{array}{l}11.70 \pm 10.11(1-53) \\
(\mathrm{n}=173)\end{array}$ & $\begin{array}{l}7.94 \pm 7.75(1-38) \\
(\mathrm{n}=801)\end{array}$ & $<0.001$
\end{tabular}

${ }^{*} \mathrm{p}<0.05$ was defined as indicative of statistical significance.

ICU, intensive care unit.

with new-onset arrhythmias in other studies. (20) Electrolyte disturbance was one of the important causes of arrhythmias in patients with acute renal dysfunction; there was a close relationship between acute renal dysfunction and arrhythmias. Electrolyte disturbance, along with systemic inflammation and a positive cumulative fluid balance, may be a possible explanation why patients who had undergone emergency surgery had increased susceptibility to arrhythmias.

For ICU patients, other factors may induce new-onset arrhythmias, such as pain, aspiration of sputum and intubation. Puncture and noise are also important factors known to induce arrhythmias, therefore, appropriate use of analgesics and sedatives is necessary to reduce the incidence of arrhythmias, especially in critically ill patients on mechanical ventilation. In addition, numerous drugs can cause electrolyte imbalance and bradyarrhythmia in critically ill patients $(21,22)$, including antiarrhythmics, antianginals, antiemetics, gastrointestinal stimulants, antibacterials, narcotics, antipsychotics, inotropes, digoxin, anesthetic agents and bronchodilators. However, in our study we were unable to demonstrate an associa- tion between the occurrence of new-onset arrhythmias and these factors.

In this study, we described the types of newonset arrhythmias in critically ill patients in two general ICUs. Atrial fibrillation is the most frequent arrhythmia in ICU patients. Although new-onset atrial fibrillation is not a cause of death, it reflects, essentially, the severity of the underlying disease and has been considered to be a marker for increased mortality and possibly a manifestation of multiple organ failure. (23) Significant ventricular arrhythmias, including ventricular tachycardia and ventricular fibrillation, occur less frequently than atrial arrhythmias, however, they may lead to a poor prognosis. New-onset arrhythmias are more likely to occur within the first 48 hours after ICU admission and are more frequent on postoperative day 2. (24)

\section{STUDY LIMITATIONS}

Some limitations must be considered for the present investigation, First of all, because of the two-center study restriction, the results of this study should be regarded as informative only. A larger, multi-center study is still warranted to investigate the characteristics of new-onset arrhythmias. What's more, the cause of arrhythmias in ICU patients also needs to be evaluated in greater detail.

\section{CONCLUSIONS}

This study analyzed the risk factors and provided an insight on the incidence and prognosis of new-onset arrhythmias in general ICU patients. New-onset arrhythmia, especially atrial fibrillation, is a very common complication in critically ill patients and is associated with an increased length of stay in ICU, higher mortality and poorer prognosis. Future studies should be focused on addressing the management of arrhythmia in the ICU, including an efficient cardiac monitoring method and prompt treatment.

\section{ACKNOWLEDGMENT}

We appreciate the technical help and support of the clinical work of Prof. Wan xian yao. 
1. Heinz G. Arrhythmias in the ICU-What do we know? Am J Respir Crit Care Med 2008;178(1):1-2.

2. Magder SA. The ups and downs of heart rate. Crit Care Med 2012;40(1): 239-45.

3. Melduni RM, Koshino Y, Shen WK. Management of arrhythmias in the perioperative setting. Clin Geriatr Med 2012;28(4):729-43.

4. Shaver CM, Chen W, Janz DR, May AK, Darbar D, Bernard GR, et al. Atrial Fibrillation Is an Independent Predictor of Mortality in Critically Ill Patients. Crit Care Med 2015;43(10):2104-11.

5. Crawford TC, Oral H. Cardiac arrhythmias: management of atrial fibrillation in the critically ill patient. Crit Care Clin 2007;23(4):85572.

6. Guenancia C, Binquet C, Laurent G, Vinault S, Bruyère R, Prin S, et al. Incidence and predictors of new-onset atrial fibrillation in septic shock patients in a medical ICU: data from 7-day Holter ECG monitoring. PLoS One. 2015;10(5):e0127168. doi: 10.1371/ journal.pone.0127168.

7. Kanji S, Williamson DR, Yaghchi BM, Albert M, McIntyre L. Epidemiology and management of atrial fibrillation in medical and noncardiac surgical adult intensive care unit patients. J Crit Care 2012;27(3): 326.e1-8.

8. Goss CH, Carson SS. Is severe sepsis associated with new-onset atrial fibrillation and stroke? JAMA 2011;306(20):2264-6.

9. Reinelt P, Karth GD, Geppert A, Heinz G. Incidence and type of cardiac arrhythmias in critically ill patients: A singal center experience in medical-cardiological ICU. Intensive Care Med 2001; 27(9):1466-73.

10. Valderrábano RJ, Blanco A, Santiago-Rodriguez EJ, Miranda C, Rivera-Del Rio Del Rio J, Ruiz J, et al. Risk factors and clinical outcomes of arrhythmias in the medical intensive care unit. J Intensive Care 2016;4:9. doi: 10.1186/s40560-016-0131-X.

11. Walkey AJ, Hogarth DK, Lip GY. Optimizing atrial fibrillation management: from ICU and beyond. Chest 2015;148(4):859-64.

12. Iscimen R, Brown DR, Cassivi SD, Keegan MT. Intensive care unit utilization and outcome after esophagectomy. J Cardioth Vascul Anesth 2010;24(3):440-6.

13. Fuchs L, Chronaki CE, Park S, Novack V, Baumfeld Y, Scott D, et al. ICU admission characteristics and mortality rates among elderly and very elderly patients. Intensive Care Med 2012;38(10):1654-61.

14. Somme D, Maillet JM, Gisselbrecht M, Novara A, Ract C, Fagon JY. Critically ill old and the oldest-old patients in intensive care: short and long term outcomes. Intensive Care Med 2003;29(12): 2137-43.

15. Walkey AJ, Laqu T, Lindenauer PK. Trends in sepsis and infection sources in the United States: a population based study. Ann Am Thorac Soc 2015;12(2):216-20.

16. Dellinger RP, Levy MM, Rhodes A, Annane D, Gerlach H, Opal SM, et al. Surviving Sepsis Campaign: international guidelines for management of severe sepsis and septic shock:2012. Intensive Care Med 2013;39(2):165-228.

17. Seemann A, Boissier F, Razazi K, Carteaux G, de Prost N, Brun-Buisson C, et al. New-onset supraventricular arrhythmia during septic shock: prevalence, risk factors and prognosis. Ann Intensive Care 2015;5(1):27.

18. Seguin P, Launey Y. Atrial fibrillation is not just an artifact in the ICU. Crit Care 2010;14(4):182.

19. Walkey AJ, Hammill BG, Curtis LH, Benjamin EJ. Long-term outcomes following development of new-onset atrial fibrillation during sepsis. Chest 2014;146(5):1187-95.

20. Schwartz A, Brotfain E, Koyfman L, Kutz R, Gruenbaum SE, Klein M, Zlotnik A. Association between hypophosphatemia and cardiac arrhythmias in the early stage of sepsis: could phosphorus replacement treatment reduce the incidence of arrhythmias? Electrolyte Blood Press 2014,12(1):19-25.

21. Doig JC. Drug-Induced Cardiac Arrhythmias. Drug Safety 1997;17(4):265-75.

22. Barnes BJ, Hollands, JM. Drug-induced arrhythmias. Crit Care Med 2010;38(6 Suppl):s188-97.

23. Walkey AJ, Greiner MA, Heckbert SR, Jensen PN, Piccini JP, Sinner MF, et al. Atrial fibrillation among medicare beneficiaries hospitalized with sepsis: incidence and risk factors. Am Heart J 2013;165(6):949-55.

24. Brathwaite D, Weissman C. The new onset of atrial arrhythmias following major noncardio thoracic surgery is associated with increased mortality. Chest 1998;114(2): 462-8. 